\title{
Dietary interventions for managing glucose abnormalities in cystic fibrosis: a systematic review protocol
}

\author{
Laura Birch $^{1 *}$ D, Fiona E. Lithander', Simon Langton Hewer ${ }^{2}$, Katie Harriman², Julian Hamilton-Shield ${ }^{1,2}$ \\ and Rachel Perry ${ }^{1}$
}

\begin{abstract}
Background: Glucose abnormalities in cystic fibrosis (CF) are common, but there is limited evidence to guide their dietary management. Progressive impaired glucose tolerance eventually leads to cystic fibrosis-related diabetes (CFRD), the most prevalent complication of CF, which is associated with increased morbidity and mortality. Optimising glycaemic control improves clinical status and reduces mortality; insulin therapy is the primary means of controlling glycaemia in CFRD, but its role in managing pre-diabetes is less clear. CF dietary therapy requires a high calorie diet due to increased energy expenditure and malabsorption, but this energy-dense diet is typically high in fat and sugar, and high sugar intakes often result in hyperglycaemia in individuals who have impaired glucose handling. Current guidelines for the dietary management of glucose abnormalities in CF are based on clinical consensus rather than empirical evidence. A systematic review conducted in 2012 on the effects of low glycaemic index dietary intervention in CF concluded that there is a dearth of evidence in this area. This review will update the systematic review by Balzer et al. in 2012 and will broaden the scope of their review to include any type of dietary intervention for managing glucose abnormalities in CF.
\end{abstract}

Methods: Quantitative studies of dietary interventions to manage glucose abnormalities in individuals aged over 5 years with CF and glucose abnormalities will be reviewed. No limits will be placed on language or study design. The comparator will be standard CF dietary therapy (energy dense, high-fat diet) in addition to insulin therapy for individuals with CFRD. Electronic databases will be searched for completed quantitative studies published in peerreview journals that focus on dietary interventions for managing glucose abnormalities in CF. Searches will be conducted from 2000 up to the present day to reflect the evolving improvements in CF management. No restrictions will be placed on study design or language. Duration of the dietary intervention must be a minimum of 2 months and only interventions in out-patient or community settings will be included. Studies must report on dietary intervention, glycaemic control, anthropometry and lung function. Evidence will be assessed for heterogeneity and a narrative review or meta-analysis conducted as appropriate.

Discussion: This systematic review will elucidate current knowledge of the effects of dietary interventions for managing glucose abnormalities in the vulnerable CF clinical population.

Systematic review registration: PROSPERO registration number: CRD42018085569 www.crd.york.ac.uk/prospero/

Keywords: Cystic fibrosis, Cystic fibrosis-related diabetes, Impaired glucose tolerance, Dietary intervention, Glycaemic control, Anthropometry, Body weight, Lung function

\footnotetext{
* Correspondence: laura.birch@bristol.ac.uk

${ }^{1} \mathrm{NIHR}$ Bristol Biomedical Research Centre - Nutrition Theme, Level 3

University Hospitals Bristol Education Centre, Upper Maudlin Street, Bristol

BS2 8AE, UK

Full list of author information is available at the end of the article
}

(c) The Author(s). 2018 Open Access This article is distributed under the terms of the Creative Commons Attribution 4.0 International License (http://creativecommons.org/licenses/by/4.0/), which permits unrestricted use, distribution, and

reproduction in any medium, provided you give appropriate credit to the original author(s) and the source, provide a link to the Creative Commons license, and indicate if changes were made. The Creative Commons Public Domain Dedication waiver (http://creativecommons.org/publicdomain/zero/1.0/) applies to the data made available in this article, unless otherwise stated. 


\section{Background}

There is limited evidence to guide dietary therapy for glucose abnormalities in cystic fibrosis (CF). CF is a chronic, life-limiting, genetic condition, characterised by abnormally thick and dehydrated secretions which lead to organ obstruction, primarily affecting the lungs and the reproductive and digestive systems [1]. Abnormalities in glucose metabolism are common and a spectrum of progressive glucose tolerance abnormalities occur as individuals grow older; indeterminate glycaemia is followed by impaired glucose tolerance (IGT), which affects $20 \%$ of 10 -year-olds and $82 \%$ of the $\mathrm{CF}$ population by the age of 30 years [2], before CF-related diabetes (CFRD) eventually develops [3]. CFRD is the most prevalent complication of CF [4], and it is a distinct form of diabetes which occurs in association with pancreatic insufficiency, primarily caused by pancreatic damage. Its pathophysiology is complex and includes the loss of pancreatic islet cells, leading to both insulin and glucagon deficiency, and fluctuating insulin resistance caused by chronic and acute inflammation and infection [4]. CFRD is typically diagnosed in late adolescence or early adulthood [5-7] and affects approximately $20 \%$ of adolescents and up to $50 \%$ of adults with CF aged over 40 years $[4,5,8]$. The early stages of CFRD is characterised by normal fasting glucose but over time fasting hyperglycaemia develops [8]. The combination of diabetes and CF leads to increased morbidity and is associated with a sixfold increase in mortality [7, 9-11]. Fewer than a quarter of people with CFRD survive beyond the age of 30 years, compared with $60 \%$ of $\mathrm{CF}$ individuals without diabetes $[12,13]$.

Optimising glycaemic control is known to improve clinical status and pulmonary function and reduce mortality [5]. Insulin therapy is the primary means of controlling glycaemia in CFRD but its role in -pre-diabetes is less clear [14]. The development of diabetes represents the onset of a second chronic disease, adding further complexity to daily CF treatment regimes. Individuals are required to monitor and control their blood glucose concentrations through regular blood glucose testing and daily insulin injections. Treating IGT in CF with insulin is controversial as it increases patient burden but it is not yet known whether early initiation of insulin reduces morbidity and mortality in the longer term [15].

In cystic fibrosis, a high energy intake is required due to increased energy expenditure and malabsorption, malnutrition risk, and gut abnormalities including delayed gastric emptying, altered intestinal motility and liver disease [7]. To date, there are no meta-analyses or randomised controlled trials of dietary intervention to manage glucose abnormalities in CF, and current guidelines are based on clinical consensus rather than empirical evidence [4, 8]. The goal of CFRD dietary management is to achieve and maintain good nutritional status and normalise blood glucose levels. There are important differences between CFRD and non-CF diabetes, which necessitate a unique approach to diagnosis and management [2]. In practice, due to the lack of specific evidence-based guidelines for managing glucose abnormalities in CF, standard CF nutritional recommendations are frequently applied [4, 8]. However, the energy-dense CF diet is typically high in fat and sugar, and high sugar intakes often results in poor glycaemic control in CF patients with glucose abnormalities. As in any clinical group, patients vary in their specific nutritional needs, and as such, dietary management must be tailored to meet these needs. As practice varies, a consistency of approach and nutritional targeting is needed in this vulnerable group.

Low glycaemic index (GI) dietary intervention has demonstrated benefits in non-CF forms of diabetes, including improved insulin sensitivity, glycaemia and quality of life in both type 1 and type 2 diabetes [16], and it is now recommended as part of their dietary management [17-19]. A systematic review conducted in 2012 to assess understanding of the effect of low GI dietary interventions in young people with CF concluded that there is a dearth of evidence in this area [20]. A scoping search of the literature has revealed that there has been little further research in this field since the 2012 review was conducted. The aim of the current review is to update the 2012 systematic review by Balzer et al. [20], to assess current understanding of glycaemic index dietary intervention in CF and to broaden the scope of their review to include any type of dietary intervention for managing glucose abnormalities in CF. Studies of dietary interventions aimed at improving glycaemic control in individuals over 5 years of age with CF and either IGT or CFRD, will be considered for inclusion. The comparator is standard CF dietary therapy (energy dense, high-fat diet), in addition to insulin therapy for individuals with CFRD. Outcomes will include glycaemic control (primary) and anthropometry and lung function (secondary).

\section{Methods}

This protocol follows the Preferred Reporting Items for Systematic Reviews and Meta-Analyses for Protocols (PRISMA-P) 2015 reporting guideline [21] (see Additional file 1). The review is registered on PROSPERO International prospective register of systematic reviews (www.crd.york.ac.uk/ prospero/); registration number: CRD42018085569. If any amendments to this protocol are required when conducting the review, these will be clearly described in the review article when prepared for publication.

\section{Eligibility criteria}

All completed, quantitative studies published in peer-review journals that focus on dietary interventions to manage glucose abnormalities in humans with CF will be included. Due to the evolving improvements in CF management, all articles published from 2000 will be screened to reflect recent care. No limits will be placed on language. 


\section{Participants}

Individuals aged over 5 years with CF and either IGT or CFRD will be included. No restrictions will be placed on upper age, gender, CF mutation or other demographics. Differences in a variety of study level characteristics may result in heterogeneity in the results (e.g. paediatric vs adult patients, good vs poor CF control, transplantation vs no transplantation). Study level characteristics (covariates) that might explain any heterogeneity will be documented and reported.

Glucose abnormalities are defined as follows:

- Oral glucose tolerance test (OGTT):

IGT:

120-min plasma glucose $7.8-11.1 \mathrm{mmol} / \mathrm{l}$

OR

Glycated haemoglobin $(\mathrm{HbA} 1 \mathrm{c}) \geq 39 \mathrm{mmol} / \mathrm{mol}$ or

$\geq 5.7 \%^{1}$

CFRD:

fasting plasma glucose concentration $\geq 7.0 \mathrm{mmol} / \mathrm{l}$

OR

120-min plasma glucose $\geq 11.1 \mathrm{mmol} / \mathrm{l}$

OR

HbA1c $\geq 48 \mathrm{mmol} / \mathrm{mol}$ or $\geq 6.5 \%$ [22]

Continuous glucose monitoring (CGM) time above

$7.8 \mathrm{mmol} / \mathrm{l} \geq 4.5 \%[23]^{2}$

All studies in all CF patients will be included, but any additional medication that may affect glycaemia will be acknowledged and if of substantial effect, analysed separately.

\section{Interventions}

Any dietary intervention for managing glucose abnormalities in CF, with or without the use of insulin therapy. Examples include low GI diets and carbohydrate counting. Only dietary interventions of a 2-month duration or more, specifically being used to manage glycaemia in CF, will be included.

\section{Comparator}

There is currently no evidence-based consensus for the dietary management of glucose abnormalities in CF, and thus, the comparators will be standard CF dietary therapy (energy dense, high-fat, high-salt diet [4]) for individuals with IGT and standard CF dietary therapy plus insulin therapy for individuals with CFRD.

\section{Setting}

Diabetes management is primarily an out-patient based speciality and therefore this review will be limited to out-patients and community settings. Any studies solely involving in-patient care will be excluded.

\section{Outcomes}

Primary outcomes are:

- Glycaemic control (OGTT, HbA1c; exploratory outcome: \% of CGM time above $7.8 \mathrm{mmol} / \mathrm{L}$ )

Secondary outcomes are:

- Body weight $(\mathrm{kg})$

- Height (m)

- BMI $\left(\mathrm{kg} / \mathrm{m}^{2}\right) /$ BMI-SDS (children \& adolescents,)

- Lung function $\left(\mathrm{FEV}_{1}, \mathrm{FVC}\right)$

- Mortality/adverse events

- Adherence and acceptability of dietary intervention

\section{Study design}

A preliminary scoping search of the literature has identified limited evidence in this area. Therefore, all study design types will be included in this review (e.g. RCTs, prospective and retrospective cohort, cross-sectional, case control, case series). Results will be presented according to study design.

\section{Information sources}

Relevant studies will be identified through a comprehensive search of the following main electronic databases: AMED, Embase, MEDLINE via OVID, Web of Science and CENTRAL via Cochrane library. To reflect the evolving improvements in CF management, articles from 2000 up to the present day will be screened, and the first 20 pages of Google Scholar. Reference lists of all included full-text articles will be hand-searched for additional original publications. OpenGrey will also be searched for any additional grey literature. Conference abstracts will be used to help identify potential studies. Conference abstracts from the following major CF and diabetes conferences will be included: International Conference of Cystic Fibrosis, North American Cystic Fibrosis Conference, European Cystic Fibrosis Society, Cystic Fibrosis Foundation Research Conference, American Diabetes Association, European Association for the Study of Diabetes, European Society of Paediatric Endocrinology, International Diabetes Federation. Study authors will be contacted to establish if full-text articles are available or to request any missing data as required.

\section{Search strategy}

A draft literature search strategy for MEDLINE database has been developed by the lead author (LB) and an experienced systematic reviewer (RP), using a combination of Medical Subject Headings (MeSH) and keyword terms. This search strategy is presented in Fig. 1. The search strategy will be revised appropriately for each database to take into account differences in controlled vocabulary 


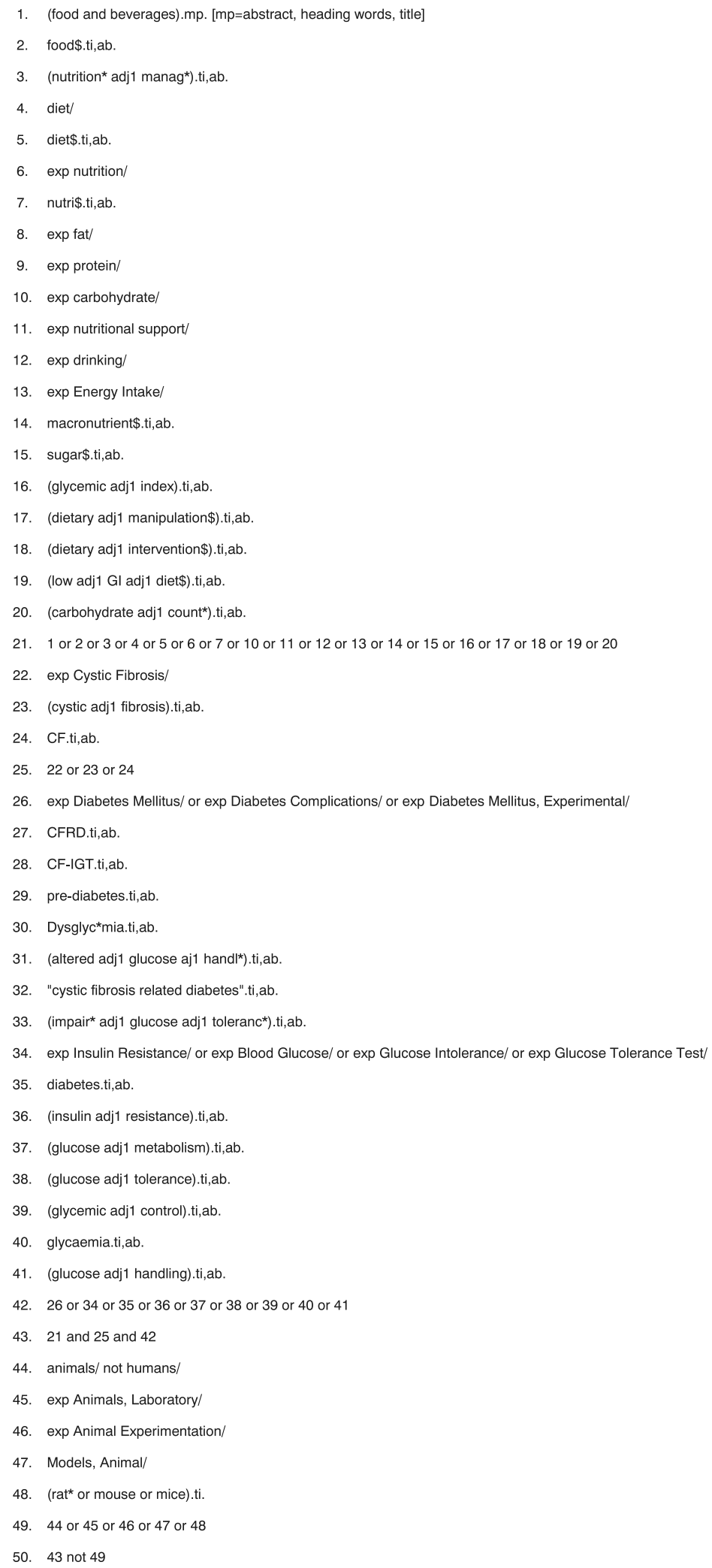

Fig. 1 Draft search strategy including planned limits (MEDLINE database) 
and syntax rules. A preliminary scoping search of Embase, MEDLINE via OVID and Web of Science databases has been conducted. The search strategy presented in Fig. 1 is a refined version of this preliminary search strategy.

\section{Data management}

EndNote X8.2 reference management software package will be used to manage all the search results throughout the review period. Covidence screening and extraction tool may also be utilised to help manage the review process.

\section{Selection process}

Titles and abstracts will be assessed for eligibility by two members of the review team (LB, RP). Articles that appear to meet the inclusion criteria will be retrieved in full and independently considered for inclusion by two members of the review team (LB, JHS). The reviewers will resolve disagreements in opinion of studies for inclusion through discussion, and the reasons for excluding studies will be recorded.

\section{Data collection process}

Data from full-text articles for inclusion will be extracted independently by two reviewers (LB, RP) using a standardised data extraction template. The template will be piloted by both reviewers before starting the review and modified as required to ensure consistency. The following data will be extracted from each study: study design; sample characteristics (size, inclusion/exclusion criteria, sex, age, socioeconomic status); dietary intervention; results (including but not limited to glycaemic control, anthropometric measures, lung function, adherence and acceptability of dietary intervention); analysis methods; study limitations; and funding sources. Disagreements in opinion of data extracted will be resolved through discussion.

\section{Risk of bias/quality assessment}

Full-text articles of all included research studies will be assessed for methodological quality independently by two members of the review team (LB, RP). The Cochrane Risk of Bias tool [24] will be used for RCTS and ROBINS-I tool [25] for observational studies. Any discrepancies between the two reviewers will be resolved through discussion or third-party adjudication. The quality of all included studies will be assessed using the Grading of Recommendations Assessment, Development and Evaluation (GRADE) system [26].

\section{Data synthesis}

A narrative review of the findings from the included studies will be presented, structured around the outcomes reported. Meta-analysis will be performed only if the identified studies are comparable. The primary outcomes of this review will be continuous variables, and we anticipate that mean values will be reported for all continuous outcomes. Results reporting categorical outcomes will be reported separately. Sub-group analysis based on age (children, adults), glucose abnormality (CFRD, IGT), CF control (good control vs poor control) and transplantation (transplant vs no transplant) will be conducted if there are sufficient numbers of comparable studies. Results will be pooled for comparable sub-sets of studies only where possible.

The preliminary scoping search conducted revealed a relatively small number of studies which appeared to be very diverse. Considerable heterogeneity between studies is therefore anticipated. Where there are sufficient studies deemed comparable, in respect of both the dietary intervention and the outcomes under consideration, their individual effect sizes will be illustrated using forest plots together with combined estimates, using random effect models. Study level characteristics (covariates) that might explain any heterogeneity, such as age, nutritional status and CF control, will be documented and reported. Sub-group analyses as outlined above will be undertaken together with meta-regression if indicated.

\section{Discussion}

This systematic review will elucidate current knowledge of the effects of dietary interventions for managing glucose abnormalities in cystic fibrosis and will highlight areas where further research is needed to inform dietary recommendations for this vulnerable clinical population.

\section{Endnotes}

${ }^{1}$ The applicability of HbA1c in the CF population is controversial due to increased red blood cell turnover [3] but will be included in this review to be as inclusive as possible.

${ }^{2}$ Exploratory outcome is not a validated definition of diabetes

\section{Additional file}

Additional file 1: PRISMA-P 2015 Checklist. PRISMA-P checklist adapted for use with systematic review protocol submissions to BioMed Central journals. (DOCX $33 \mathrm{~kb}$ )

\section{Abbreviations}

CF: Cystic fibrosis; CFRD: Cystic fibrosis-related diabetes; Gl: Glycaemic index; IGT: Impaired glucose tolerance

\section{Funding}

This review is funded by the National Institute of Health Research (NIHR) as part of a Clinical Doctoral Research Fellowship (C-DRF) award held by the lead author (LB). All co-authors are funded through the NIHR Bristol Biomedical Research Centre-Nutrition Theme. 


\section{Disclaimer}

This is an independent opinion from a Biomedical Research Centre in the National Institute for Health Research Biomedical Research Centre and Unit Funding Scheme. The views expressed in this publication are those of the authors and not necessarily those of the National Health Service, the National Institute for Health Research or the Department of Health.

\section{Authors' contributions}

LB conceived of this systematic review and wrote the protocol manuscript. $\mathrm{SLH}, \mathrm{KH}$ and JHS provided expertise in relation to the clinical parameters of this review. FL participated in the design of the study and helped to draft the manuscript. RP provided methodological advice and assistance in relation to conducting the systematic review. All authors have read and approved the final protocol manuscript.

\section{Authors' information}

$\mathrm{LB}$ is a research dietitian at the NIHR Bristol Biomedical Research Centre-Nutrition Theme. All co-authors are members of either the NIHR Bristol Biomedical Research Centre-Nutrition Theme or the CF Specialist Centre at the Bristol Royal Hospital for Children. FL is a senior research associate and dietitian. $\mathrm{SLH}$ is a consultant in Paediatric Respiratory Medicine. KH is a paediatric CF dietitian. JHS is a professor of Diabetes and Metabolic Endocrinology. RP is a systematic reviewer.

\section{Ethics approval and consent to participate}

Not applicable as this is a systematic review of completed studies.

\section{Consent for publication}

Not applicable

\section{Competing interests}

The authors declare that they have no competing interests.

\section{Publisher's Note}

Springer Nature remains neutral with regard to jurisdictional claims in published maps and institutional affiliations.

\section{Author details}

${ }^{1}$ NIHR Bristol Biomedical Research Centre - Nutrition Theme, Level 3 University Hospitals Bristol Education Centre, Upper Maudlin Street, Bristol BS2 8AE, UK. ${ }^{2}$ CF Specialist Centre, Bristol Royal Hospital for Children, Upper Maudlin Street, Bristol BS2 8BJ, UK.

Received: 16 January 2018 Accepted: 19 June 2018

Published online: 18 July 2018

\section{References}

1. Filbrun AG, Lahiri T, Ren CL. Handbook of cystic fibrosis. Switzerland: Springer International Publishing; 2016.

2. Bismuth $\mathrm{E}$, et al. Glucose tolerance and insulin secretion, morbidity, and death in patients with cystic fibrosis. J Pediatr. 2008;152(4):540-5.

3. Bridges N. Diabetes in cystic fibrosis. Paediatr Respir Rev. 2013;14(Suppl 1): $16-8$.

4. Moran A, et al. Management of cystic fibrosis-related diabetes in children and adolescents. Pediatr Diabetes. 2014;15(Suppl 20):65-76.

5. Moran A, et al. Cystic fibrosis-related diabetes: current trends in prevalence, incidence, and mortality. Diabetes Care. 2009;32(9):1626-31.

6. Lanng $S$, et al. Glucose tolerance in patients with cystic fibrosis: five year prospective study. BMJ. 1995;311(7006):655-9.

7. Rodman HM, Doershuk CF, Roland JM. The interaction of 2 diseases: diabetes mellitus and cystic fibrosis. Medicine. 1986;65(6):389-98.

8. Castellani $\mathrm{C}$, et al. ECFS best practice guidelines: the 2018 revision. J Cyst Fibros. 2018;17(2):153-78

9. Rosenecker J, et al. Diabetes mellitus and cystic fibrosis: comparison of clinical parameters in patients treated with insulin versus oral glucoselowering agents. Pediatr Pulmonol. 2001;32(5):351-5.

10. Wilson $\mathrm{D}$, et al. Challenges in the dietary treatment of cystic fibrosis related diabetes mellitus. Clin Nutr. 2000;19(2):87-93.

11. Chamnan $\mathrm{P}$, et al. Diabetes as a determinant of mortality in cystic fibrosis. Diabetes Care. 2010;33(2):311-6.
12. Mohan $\mathrm{K}$, et al. Mechanisms of glucose intolerance in cystic fibrosis. Diabet Med. 2009:26(6):582-8.

13. Finkelstein $\mathrm{SM}$, et al. Diabetes mellitus associated with cystic fibrosis. J Pediatr. 1988:112(3):373-7.

14. Rana M, et al. Cystic fibrosis-related diabetes in children-gaps in the evidence? Nat Rev Endocrinol. 2010;6(7):371-8.

15. White $\mathrm{H}$, et al. Nutritional decline in cystic fibrosis related diabetes: the effect of intensive nutritional intervention. J Cyst Fibros. 2009:8(3):179-85.

16. Thomas D, Elliott EJ. Low glycaemic index, or low glycaemic load, diets for diabetes mellitus. Cochrane Libr. 2009. https://doi.org/10.1002/14651858. CD006296.pub2

17. Craig M, et al. National evidence-based clinical care guidelines for type 1 diabetes in children, adolescents and adults, vol. 346. Canberra: Australian Government Department of Health and Ageing; 2011.

18. American Diabetes Association. 4. Lifestyle management: standards of medical Care in Diabetes-2018. Diabetes Care. 2018;41 (Supplement 1):S38-50.

19. Dyson $\mathrm{P}$, et al. Diabetes UK evidence-based nutrition guidelines for the prevention and management of diabetes. Diabet Med. 2011;28(11):1282-8.

20. Balzer BW, et al. Low glycaemic index dietary interventions in youth with cystic fibrosis: a systematic review and discussion of the clinical implications. Nutrients. 2012;4(4):286-96.

21. Moher $D$, et al. Preferred reporting items for systematic review and metaanalysis protocols (PRISMA-P) 2015 statement. Syst Rev. 2015;4(1):1.

22. American Diabetes Association. 2. Classification and diagnosis of diabetes: standards of medical care in Diabetes_-2018. Diabetes Care. 2018; 41(Supplement 1):S13-27.

23. Hameed $\mathrm{S}$, et al. Early glucose abnormalities in cystic fibrosis are preceded by poor weight gain. Diabetes Care. 2010;33(2):221-6.

24. Higgins JP, et al. The Cochrane Collaboration's tool for assessing risk of bias in randomised trials. BMJ. 2011;343:d5928.

25. Sterne JA, et al. ROBINS-I: a tool for assessing risk of bias in non-randomised studies of interventions. BMJ. 2016;355:44919.

26. Guyatt $\mathrm{GH}$, et al. GRADE guidelines: a new series of articles in the journal of clinical epidemiology. J Clin Epidemiol. 2011;64(4):380-2.

\section{Ready to submit your research? Choose BMC and benefit from:}

- fast, convenient online submission

- thorough peer review by experienced researchers in your field

- rapid publication on acceptance

- support for research data, including large and complex data types

- gold Open Access which fosters wider collaboration and increased citations

- maximum visibility for your research: over $100 \mathrm{M}$ website views per year

At BMC, research is always in progress.

Learn more biomedcentral.com/submissions 